\title{
ESTRATEGIAS PARA MEJORAR LA SEGURIDAD DEL METRO LIGERO A TRAVÉS DE SU INSERCIÓN URBANA
}

\author{
Margarita Novales Ordax \\ Profesora Titular de Universidad, Grupo de Ferrocarriles y Transportes, \\ Universidad de A Coruña \\ Andrés Muñoz de Dios \\ Director Gerente, Metro de Tenerife \\ Joan Carsi Pérez ${ }^{1}$, Olatz Ortiz Etxeberria ${ }^{2}$ \\ ${ }^{1}$ Director General Adjunto, ${ }^{2}$ Responsable de Estudios y Proyectos, \\ Tramvia Metropolitá S.A.
}

\section{RESUMEN}

La COST Action 1103: "Operation and safety of tramways in interaction with public space", desarrollada entre 2011 y 2015, tenía como objetivo alcanzar un mejor entendimiento de la seguridad del metro ligero en su relación con el espacio urbano.

Durante la Acción se puso de manifiesto que los diferentes sistemas de metro ligero europeos tienen filosofías totalmente distintas entre ellos. Algunas redes prestan más atención a la protección del sistema, con un planteamiento cercano al del ferrocarril convencional. Otras tratan de conseguir sistemas mejor integrados con la ciudad, facilitando la coexistencia con peatones y ciclistas en el centro urbano, pero garantizando en cualquier caso la capacidad y velocidad del metro ligero en el área metropolitana.

En cualquier caso, a pesar de las particularidades de cada red, los metros ligeros de todo el mundo se enfrentan a retos de seguridad similares, y las soluciones aplicadas en un lugar pueden ser útiles para otras redes de nueva implantación o existentes que se enfrenten a los mismos problemas.

En esta ponencia se presentarán algunas de las medidas utilizadas por diferentes metros ligeros europeos para mejorar la seguridad de ciertos puntos diagnosticados previamente como puntos conflictivos (a través de los datos de accidentes de los operadores). El origen de los datos utilizados es un cuestionario sobre dichos puntos conflictivos realizado durante la Acción, que fue respondido por 24 operadores de toda Europa e Israel. 


\section{INTRODUCCIÓN}

La COST (European Cooperation in Science and Technology) Action TU1103, "Operation and safety of tramways in interaction with public space", se centró en el incremento de la seguridad de los sistemas de metro ligero (LRT) a través de una mejor organización y diseño de su inserción en el espacio urbano. En la Acción participaron 34 entidades que incluyen operadores de metro ligero, entidades de gestión de la seguridad y organismos de investigación de 14 países europeos diferentes (además de Israel), incluyendo a la UITP (Unión Internacional del Transporte Público).

En los sistemas de metro ligero existen dos partes fundamentales que influyen en la seguridad: la infraestructura y la gestión de la operación. La infraestructura constituye la parte más cara, y realizar cambios posteriores a su construcción resulta costoso y complejo. Por su parte, la gestión de la operación puede resolver algunos problemas generados por un diseño deficiente de la infraestructura, pero esta capacidad es limitada y no se puede aplicar a todos los problemas infraestructurales.

Los principales problemas de seguridad a los que se enfrentan los sistemas de metro ligero están relacionados, en general, con la interacción con el resto de usuarios del espacio urbano. Esta interacción se concentra normalmente en torno a las intersecciones con vehículos de carretera (intersecciones y glorietas), y a los pasos de peatones u otros puntos en los que éstos cruzan las vías. En esta ponencia se mostrarán varias medidas aplicadas para mejorar la seguridad en ciertos puntos conflictivos, algunas de ellas basadas en mejoras de la infraestructura, y otras en cambios en la gestión de la operación.

\section{SEGURIDAD Y DISEÑO DEL METRO LIGERO}

En el diseño del metro ligero se deben considerar tres aspectos fundamentales para garantizar la seguridad: la visibilidad entre el metro ligero y el resto de usuarios del espacio urbano (conductores de los vehículos de carretera, peatones y ciclistas); la percepción del sistema (e información al resto de usuarios del espacio urbano); y la protección del metro ligero en su interacción con ellos.

El primer aspecto, la visibilidad, es esencial para garantizar la seguridad del metro ligero. Durante el diseño del trazado, la visibilidad debe tenerse siempre en cuenta para evitar problemas posteriores. En general, las consideraciones fundamentales sobre visibilidad son:

- En lo que se refiere a la visibilidad en las intersecciones, la mejor forma de garantizarla es hacer que las calles que confluyen en la intersección sean perpendiculares entre ellas. Incluso si no lo eran previamente a la construcción del sistema de metro ligero, a veces se puede transformar la intersección haciéndola perpendicular con algunas pequeñas modificaciones, si existe espacio disponible para ello (Figuras 1a y 1b).

- Adicionalmente, tanto en las intersecciones como fuera de ellas se deben eliminar o recolocar, si es posible, los elementos que limiten o impidan la visibilidad entre el metro ligero y el resto de usuarios del espacio urbano (Figuras 1a y 1b). Por otra parte, se debe 
considerar la iluminación necesaria para garantizar la visibilidad de noche.

- También se debe tener en cuenta la visibilidad de las señales y semáforos. En secciones posteriores se presentarán diferentes medidas relacionadas con este tema.

En cualquier caso, es habitual que existan ciertas zonas de la red de metro ligero en las que la visibilidad no es adecuada debido a la geometría de las vías (curvas horizontales o verticales de radio reducido), al tipo de calles (anchura limitada), o a la existencia de obstáculos que no se pueden eliminar o recolocar. En estos casos, la falta de visibilidad suficiente se debe mitigar potenciando las medidas de percepción y protección del metro ligero.

Entre las medidas relacionadas con la percepción, las más importantes en lo que se refiere al diseño de la infraestructura son: acabado de las vías con diferente material, color o textura que las zonas adyacentes (Figuras 1b y 1c); y marcado del área de barrido del vehículo. Las medidas relacionadas con la percepción en el diseño del vehículo son: utilización de colores llamativos en los vehículos de metro ligero permitiendo una buena identificación en el paisaje urbano; circulación del metro ligero con las luces encendidas; y utilización de señales sonoras de aviso en puntos específicos para anunciar que el vehículo se está acercando.

Adicionalmente, para mejorar la percepción se pueden utilizar diferentes medidas informativas, como señales informativas verticales y pintura horizontal, del tipo de la que se utiliza en los pasos de peatones (Figura 2a).

Finalmente, es muy importante la protección del metro ligero respecto al resto de usuarios del espacio urbano, tanto para garantizar la seguridad como para permitir al metro ligero circular a velocidades adecuadas en ciertas zonas específicas. Entre las medidas de protección están las barreras físicas (como separadores respecto al tráfico de carretera, o vallas y barreras para peatones, del tipo de los que se muestran en las Figuras 1c y $2 \mathrm{~b}-$ véanse medidas adicionales en Novales, Teixeira y Fontaine, 2014 -), así como las señales de reglamentación (prescriptivas) y los semáforos para vehículos de carretera (Figura 1d). 


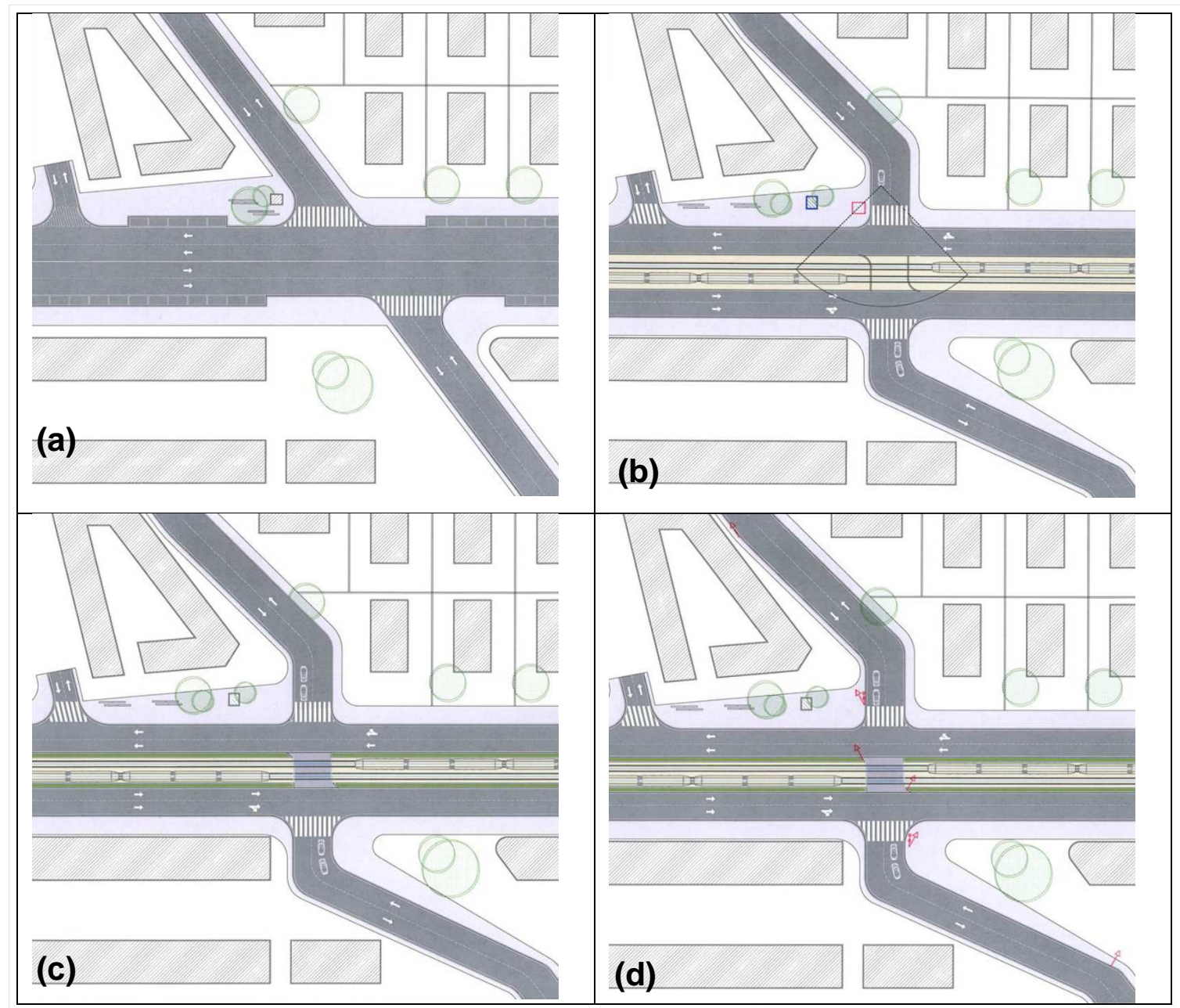

Fig. 1 - Medidas para visibilidad, percepción y protección respecto a los vehículos de carretera.

a) Situación de la intersección antes de la implementación del metro ligero.

b) Visibilidad: transformación a intersección perpendicular; eliminación de obstáculos (círculos verdes y reposicionamiento de la posición roja a la azul) + Percepción: pavimento diferenciado en las vías de metro ligero.

c) Percepción: identificación de la zona de cruce por contraste de color + Protección: separador vegetal.

d) Protección: señales verticales (a larga y corta distancia) + Semáforos (cuando los semáforos no funcionan las señales marcan la prioridad). 


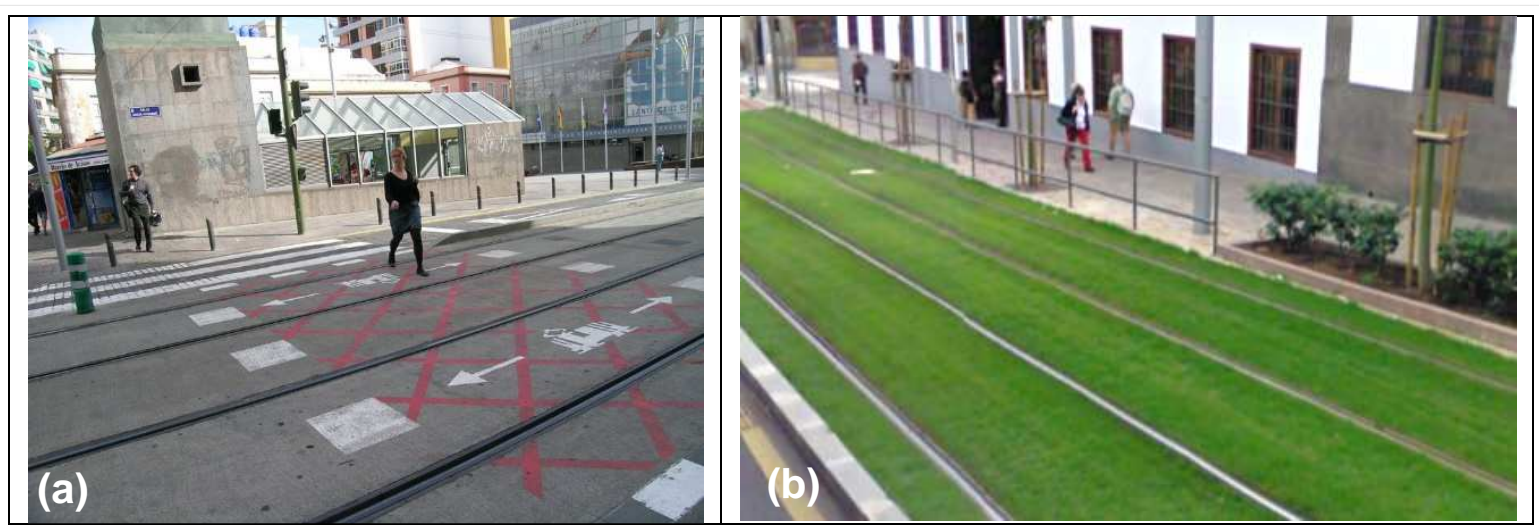

Fig. 2 - Medidas para percepción (información) y protección respecto a los peatones.

a) Percepción (información): pintura horizontal.

b) Protección: barreras (metálica y vegetal).

\section{METODOLOGÍA}

Durante la Acción COST se envió un cuestionario a diversos operadores de metro ligero en el que se les pedía que identificasen los principales puntos conflictivos ("hotspots") de su red, así como los que han dejado de serlo por las diferentes medidas aplicadas.

En el cuestionario participaron un total de 24 operadores de 13 países diferentes: Austria (Viena); Bélgica (Bruselas); República Checa (Brno, Praga, Olomouc); Francia (Le Mans, Lyon, Montpellier); Alemania (Berlín, Bremen); Irlanda (Dublín); Israel (Jerusalén); Italia (Milán); Países Bajos (Ámsterdam); Portugal (Lisboa, Oporto); España (Barcelona, Bilbao, Tenerife); Suiza (Berna, Ginebra, Zürich); y Reino Unido (Manchester, Sheffield).

La definición dada para los hotspots en el cuestionario fue: "las zonas o puntos del espacio urbano donde se produjo un mayor número de accidentes (colisiones) en un período determinado". Los antiguos hotspots son las zonas o puntos de la red que eran puntos conflictivos en el pasado pero han dejado de serlo debido a las medidas implementadas para mejorar la situación.

En el punto siguiente de esta ponencia se presentará una revisión de algunos de los antiguos puntos conflictivos y las medidas aplicadas por los operadores. El objetivo es que otros operadores que se enfrenten a problemas similares, o que estén diseñando una nueva línea, puedan utilizar estos ejemplos como buenas prácticas para mejorar sus redes. En los casos en que sea posible, se tratará de respaldar la eficiencia de estas medidas con el dato del número de accidentes por año antes y después de su implementación.

Además del cuestionario sobre puntos conflictivos, durante la Acción se realizó otro cuestionario sobre buenas y malas prácticas para el diseño de los puntos de interacción, entendidos como los principales puntos o zonas de la infraestructura del metro ligero cuyo diseño debe estudiarse en detalle para garantizar la seguridad del sistema en su interacción con el espacio urbano (Novales, Teixeira y Fontaine, 2014). En este cuestionario se reunieron más de 130 ejemplos, y en esta ponencia se incluye también un breve resumen de 
algunas de las conclusiones obtenidas del mismo.

En cualquier caso, todos los ejemplos de antiguos hotspots, de los puntos de interacción, así como información adicional sobre la Acción COST, se pueden encontrar en el informe final de la misma (Fontaine et al., 2015).

\section{ANTIGUOS PUNTOS CONFLICTIVOS Y BUENAS PRÁCTICAS}

En esta ponencia se presenta un total de 9 antiguos puntos conflictivos situados en glorietas (5) y en otro tipo de intersecciones (4). Adicionalmente, en la última sección de cada apartado se incluye información suplementaria sobre algunas de las conclusiones del cuestionario sobre puntos de interacción realizado durante la Acción.

\subsection{Intersecciones}

Existen diferentes publicaciones sobre intersecciones para tráfico de carretera y metro ligero. En el TCRP Report 69 del TRB (Korbe et al., 2001) se presentan diferentes tratamientos para intersecciones en las que el metro ligero circula a más de $55 \mathrm{~km} / \mathrm{h}$. Por su parte, Pecheux y Golembiewski (2011) realizan un estudio sobre la comprensión de la señalización en intersecciones con metro ligero. Otros documentos como el TCRP Synthesis 79 del TRB (Pecheux y Saporta, 2009), Farrán (2000) o Coifman y Bertini (1997) estudian diferentes medidas para mejorar la seguridad en intersecciones de metro ligero con giro a la izquierda para vehículos de carretera, o para evitar la realización de giros a la izquierda en lugares donde éstos están prohibidos. Finalmente, en Currie y Reynolds (2011) se tratan los "giros en anzuelo" ("hook-turns") y giros a la derecha para el caso australiano (en donde se circula por la izquierda).

A continuación se presentan algunos de los antiguos puntos conflictivos en intersecciones recopilados en el cuestionario de la Acción.

\subsubsection{Intersección Blackhall Place - Benburb Street, Dublín (Irlanda)}

Se trata de una intersección entre una calle de sentido único para el tráfico de carretera con doble vía lateral para el metro ligero (Benburb Street), con una calle de dos sentidos de circulación (Blackhall Place). La velocidad del metro ligero en esta zona es de $25 \mathrm{~km} / \mathrm{h}$. En este punto se han producido varios accidentes debido a infracciones de tipo paso del semáforo en rojo por parte de los conductores de los vehículos de carretera. Desde el año 2009 se han implementado diversas medidas (Figuras 3):

- Para mejorar la visibilidad de las señales: instalación de señales avanzadas de advertencia de la presencia del metro ligero de mayor tamaño del habitual (900 mm) a ambos lados de la intersección.

- Para mejorar la percepción: instalación de un pavimento con color en contraste en las calles de aproximación a la intersección.

- Para mejorar la protección: instalación de luces encastradas en el pavimento que parpadean en color rojo en combinación con la luz roja del semáforo. Estas luces se 
instalaron en las calles de aproximación a la intersección a ambos lados de la misma. En Tydlacka, Voigt y Langford (2011) se trata la eficacia de una solución similar aplicada en Houston, Texas (Estados Unidos).

- Para mejorar la protección: implementación de un pavimento antideslizante de alta adherencia, también a ambos lados de la intersección, para mejorar la capacidad de frenado de los vehículos de carretera ante el semáforo en rojo.

- Para reducir la probabilidad de que se produzca un accidente, incluso ante infracciones por parte de los conductores de los vehículos de carretera, el vehículo de metro ligero debe detenerse antes de atravesar esta intersección. Esta no es una medida que se pueda utilizar de manera generalizada, ya que reduce la velocidad comercial del metro ligero, y por tanto la calidad de servicio del mismo.

- Una medida que se plantea a futuro para mejorar la protección, y que ya se ha aplicado en otras intersecciones en Dublín, consiste en la instalación de una cámara de infracción del tipo paso del semáforo en rojo, para multar automáticamente a los conductores que cometan estas infracciones en esta intersección. Esta solución también se utiliza en el metro ligero de Los Ángeles (Korbe et al., 2001).

Desde la implementación de estas medidas (a partir del año 2009 en adelante), el número de accidentes parece haberse reducido ligeramente, aunque los datos no son concluyentes. El número de accidentes por año en este punto fue: año 2004: 3 accidentes; año 2005: 3; 2006: 2; 2007: 0; 2008: 2; 2009: 1; 2010: 4; 2011: 1; 2012: 1; 2013: 0; 2014: 2. 


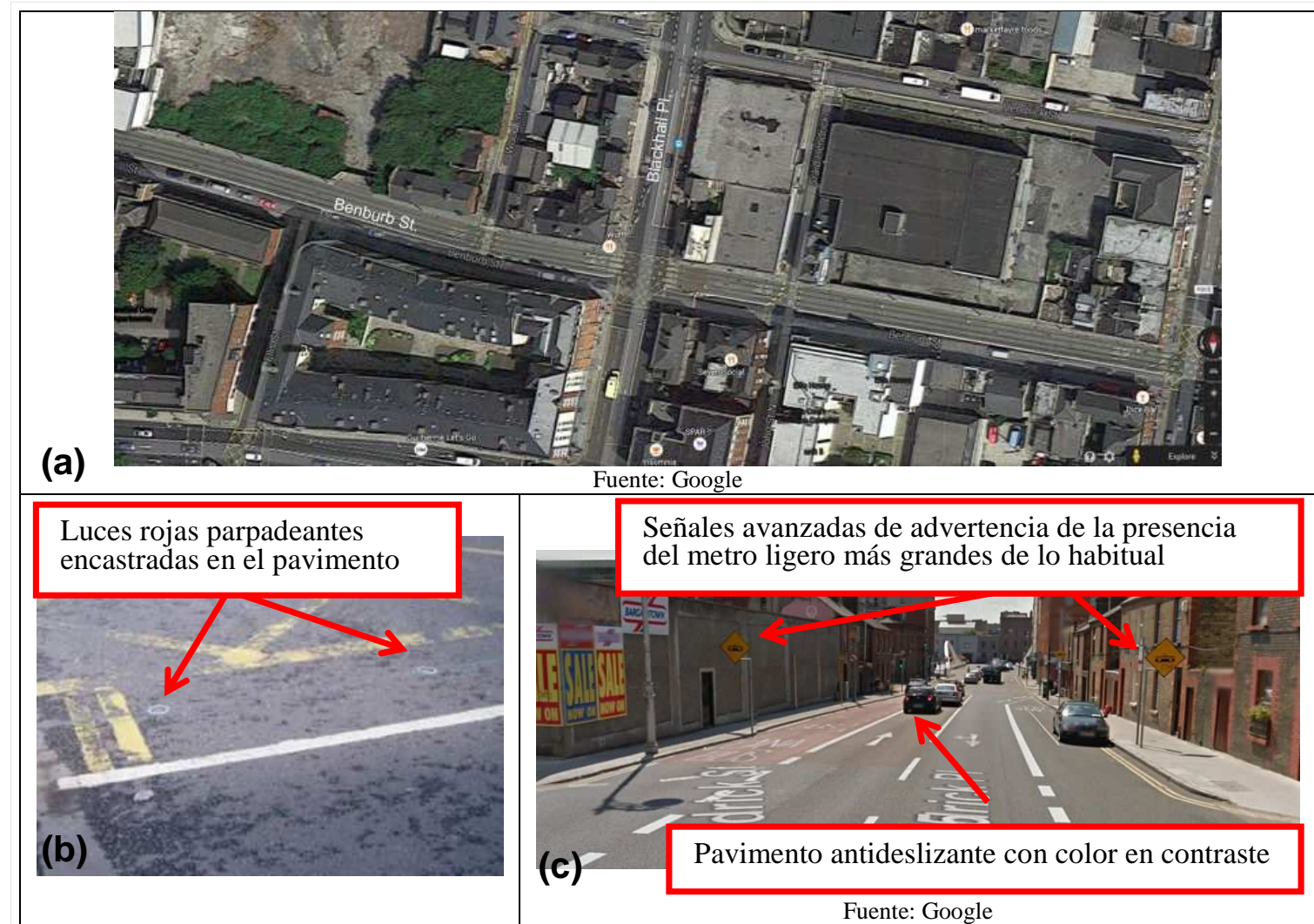

Fig. 3 - Antiguo punto conflictivo en la intersección Blackhall Place - Benburb Street: evitar las infracciones de tipo paso de semáforo en rojo.

a) Situación del punto conflictivo.

b) Luces rojas parpadeantes encastradas en el pavimento.

c) Señal avanzada de advertencia de la presencia del metro ligero con tamaño mayor del habitual y pavimento antideslizante con color en contraste.

\subsubsection{Intersección de Bow Street, Dublín (Irlanda)}

Se trata de una intersección entre una calle muy estrecha (Bow) y otra calle estrecha por la que sólo circula el metro ligero. Tanto el metro ligero como el tráfico de carretera están controlados por semáforos. En esta intersección existía un importante problema de intervisibilidad entre los vehículos de metro ligero y de carretera, debido a la existencia de una valla sólida de madera a mano derecha, con el resultado de accidentes debido a que los conductores de vehículos de carretera no veían al vehículo de metro ligero y pasaban el semáforo en rojo.

La medida aplicada para resolver este problema consistió simplemente en reemplazar la valla de madera por una de tipo malla (Figura 4).

Adicionalmente, para mejorar la protección se modificó el ciclo semafórico de manera que en la actualidad el semáforo se encuentra siempre en rojo hasta que el controlador es activado por la presencia de un vehículo de carretera (que se detecta mediante un lazo en la zona del semáforo). De esta manera, los conductores de vehículos de carretera siempre se tienen que detener en la intersección, mejorando su posibilidad de reacción ante la presencia del vehículo de metro ligero, porque siempre estarán avanzando a velocidad reducida al estar 
arrancando.

Desde la implementación de estas medidas (en octubre de 2012), el número de accidentes parece haberse reducido ligeramente, aunque los datos no son concluyentes. El número de accidentes por año en este punto fue: año 2004: 0 accidentes; año 2005: 1; 2006: 0; 2007: 1; 2008: 1; 2009: 1; 2010: 0; 2011: 3; 2012: 1; 2013: 1; 2014: 0.

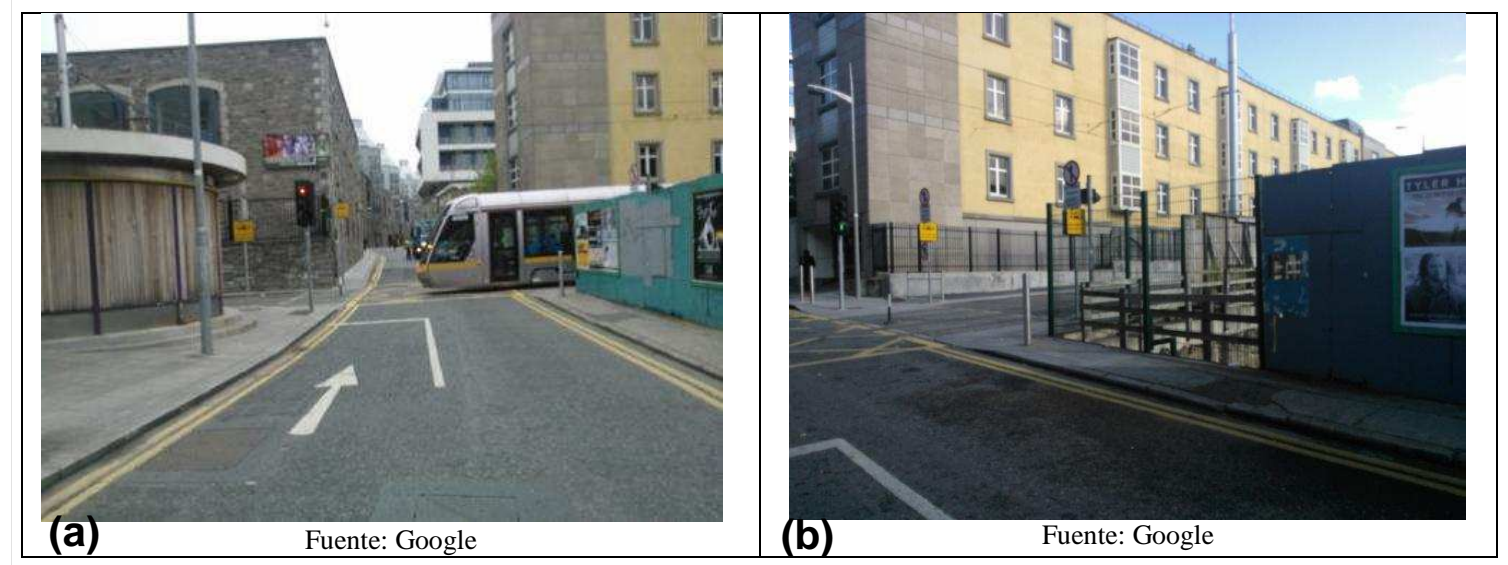

Fig. 4 - Antiguo punto conflictivo en la intersección de Bow Street: mejora de visibilidad mediante la transformación de una valla sólida.

a) Situación antes de la actuación.

b) Situación después de la actuación.

\subsubsection{Ringstrasse en Dübendorf, Zürich (Suiza)}

El problema en este caso era la deficiente percepción de las vías del metro ligero en una intersección de una nueva línea tras su puesta en explotación. Las vías en doble dirección del metro ligero se encuentran en el lateral de la calle (Figura 5a), y los conductores de vehículos de carretera que entraban en esta calle desde la perpendicular no percibían la existencia de las vías, especialmente con presencia de nieve, y se quedaban atrapados en ellas debido al acabado de vía en césped en la primera zona y de vía en placa en la zona posterior sobre el puente. Durante los tres primeros meses tras la inauguración de esta línea se produjeron 6 eventos de este tipo.

Para reforzar la percepción en esta intersección se tomaron diversas medidas: pintado de señalización horizontal adicional en el pavimento; disposición de una nueva señal de tráfico indicando el camino correcto a los conductores de carretera; instalación de un bolardo entre las vías de cada sentido de circulación; y, finalmente, el posterior pintado del pavimento de la zona de vías de la intersección en verde (Figura 5b). Gracias a estas medidas se ha erradicado la invasión de las vías en este punto. 


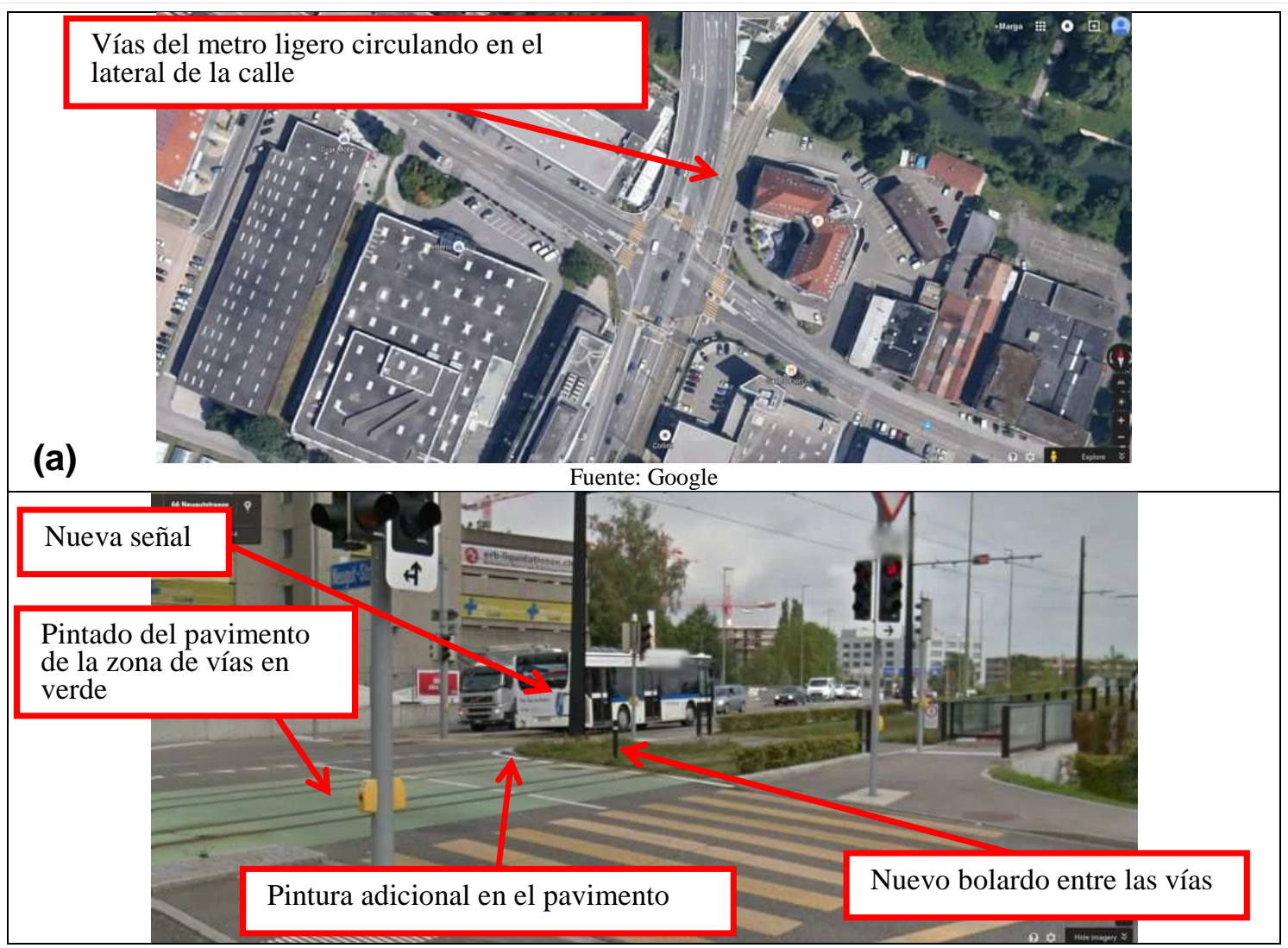

Fig. 5 - Antiguo punto conflictivo en la intersección de Ringstrasse en Dübendorf: mejora de la percepción.

a) Disposición de la intersección.

b) Medidas implementadas.

\subsubsection{Intersección Ordsall Lane - Exchange Quays, Manchester (Reino Unido)}

En este punto conflictivo existen dos intersecciones muy cercanas entre sí (distancia de alrededor de $70 \mathrm{~m}$ ), ambas controladas mediante semáforos (Figura 6). En algunas ocasiones, los conductores que se encuentran en la intersección de Ordsall Lane se fijan en los semáforos de la intersección siguiente (Trafford Road) en verde, lo que les lleva a confusión de manera que cruzan su intersección con el metro ligero estando su semáforo en rojo. Entre 2003 y 2013 se produjeron 14 incidentes de este tipo.

La solución implementada consiste en la mejora de la protección equipando los semáforos de la intersección de Trafford Road con lamas que evitan el problema de que se vean a distancia sus luces. De esta manera, los conductores sólo pueden ver la luz roja o verde de este semáforo cuando se encuentran próximos al mismo.

La situación parece haber mejorado desde la implementación de esta medida. 


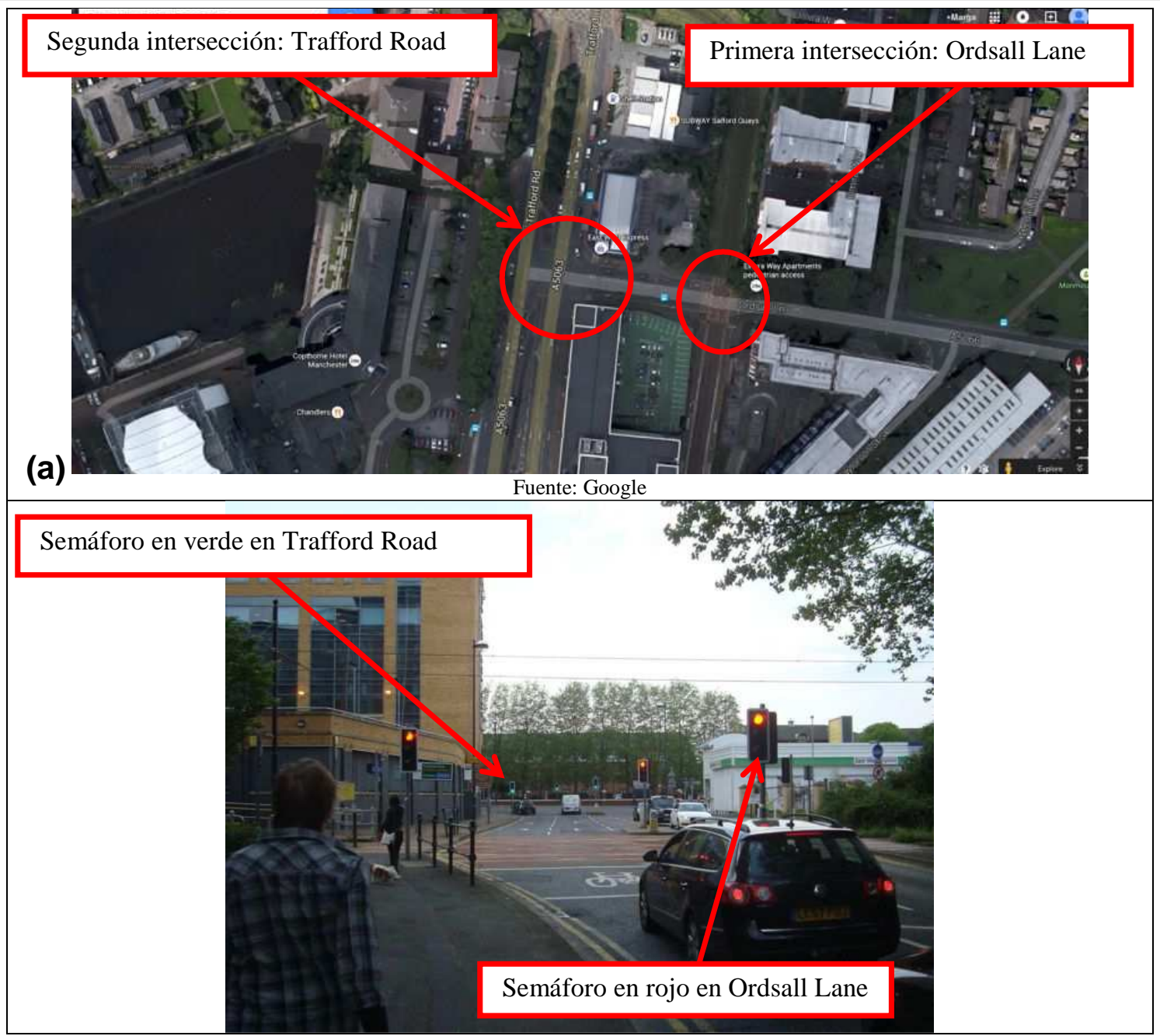

Fig. 6 - Antiguo punto conflictivo en la intersección Ordsall Lane - Trafford Road: mejora de la protección.

a) Disposición de la intersección.

b) Problema de visión a distancia de los semáforos de Trafford Road.

\subsubsection{Otras medidas para mejorar la seguridad en las intersecciones}

Algunas de las medidas para mejorar la seguridad en las intersecciones mencionadas en el cuestionario sobre puntos de interacción, adicionales a las presentadas en los puntos anteriores, son:

- Para mejorar la visibilidad en caso de giros a la izquierda: diseño del giro a la izquierda perpendicularmente a las vías de metro ligero. Para ello, se puede introducir una zona verde entre el carril de giro y las vías, permitiendo a los conductores de los vehículos de carretera realizar el giro antes de cruzar las vías, de manera que se las encuentren en perpendicular, por lo que tendrán una buena visibilidad del vehículo de metro ligero que se acerca a la intersección, en su caso (Novales, Teixeira y Fontaine, 2014).

- Para mejorar la percepción y protección: disposición en los carriles de circulación para vehículos de carretera de una suave rampa justo antes de alcanzar la intersección, para ponerse a cota con las vías del metro ligero (algo más elevadas que la calzada), de manera que se aumenta la atención de los conductores a la vez que se consigue una reducción de velocidad; refuerzo de los semáforos incluyendo un símbolo de metro ligero activo que 
empieza a parpadear cuando el vehículo de metro ligero se está acercando; hacer el giro a la izquierda físicamente imposible o muy complicado (Novales, Teixeira y Fontaine, 2014); utilización de barreras para el tráfico (con líneas rojas y blancas) coordinadas con los semáforos y con la detección de aproximación del vehículo de metro ligero (esta medida sólo se recomienda para puntos especialmente problemáticos).

\subsection{Glorietas}

En general, cuando se introduce un sistema moderno de metro ligero en una glorieta, éste circula por su centro y el funcionamiento de la misma cambia. La glorieta funciona como una glorieta convencional cuando no está presente ningún vehículo de metro ligero, pero se instalan semáforos al menos en los puntos en que el vial circular cruza las vías, para darle prioridad a los vehículos de metro ligero cuando se aproximan a la glorieta (Novales, Teixeira y Fontaine, 2014). Para evitar accidentes en este tipo de configuraciones, además de garantizar una visibilidad adecuada, se deben reforzar la percepción y protección, como se muestra en los ejemplos que se presentan a continuación.

La perspectiva francesa respecto a las glorietas con metro ligero se presenta en las recomendaciones francesas (CERTU, 2008).

\subsubsection{Glorieta de Paul Cézanne, Le Mans (Francia)}

El metro ligero de Le Mans se inauguró en noviembre de 2007. Este metro ligero circula por el centro de la glorieta de Paul Cézanne y se han producido varios accidentes con vehículos de carretera por violación por parte de sus conductores de los semáforos en rojo y la prioridad del metro ligero. También se han producido accidentes de tipo caída de pasajeros dentro del vehículo por los frenados de emergencia realizados como consecuencia de esta circunstancia.

Las medidas aplicadas para solucionar este problema fueron las siguientes (Figura 7):

- Mejora de la percepción introduciendo marcas tipo "dientes de tiburón" en los puntos en los que el vial circular de la glorieta cruza las vías del metro ligero. Esta medida se implementó en septiembre de 2010.

- Mejora de la protección introduciendo un segundo semáforo de tipo R24, que parpadea alternativamente con el ya existente. Esta medida se implantó en mayo de 2012.

La evolución del número de incidentes (accidentes y caídas de pasajeros) en este punto es la siguiente: noviembre de 2007 a final de 2008: 7; año 2009: 2; año 2010: 0; año 2011: 1; 2012: 2; 2013: 0. La reducción rápida del número de accidentes tras el comienzo de la explotación es bastante común en la implementación de nuevas redes de metro ligero, ya que el resto de usuarios del espacio urbano necesitan un tiempo de aprendizaje para saber cómo interactuar con el nuevo sistema. En cualquier caso, parece que se ha producido una ligera mejora debido a las medidas implementadas, aunque los datos no son concluyentes. 


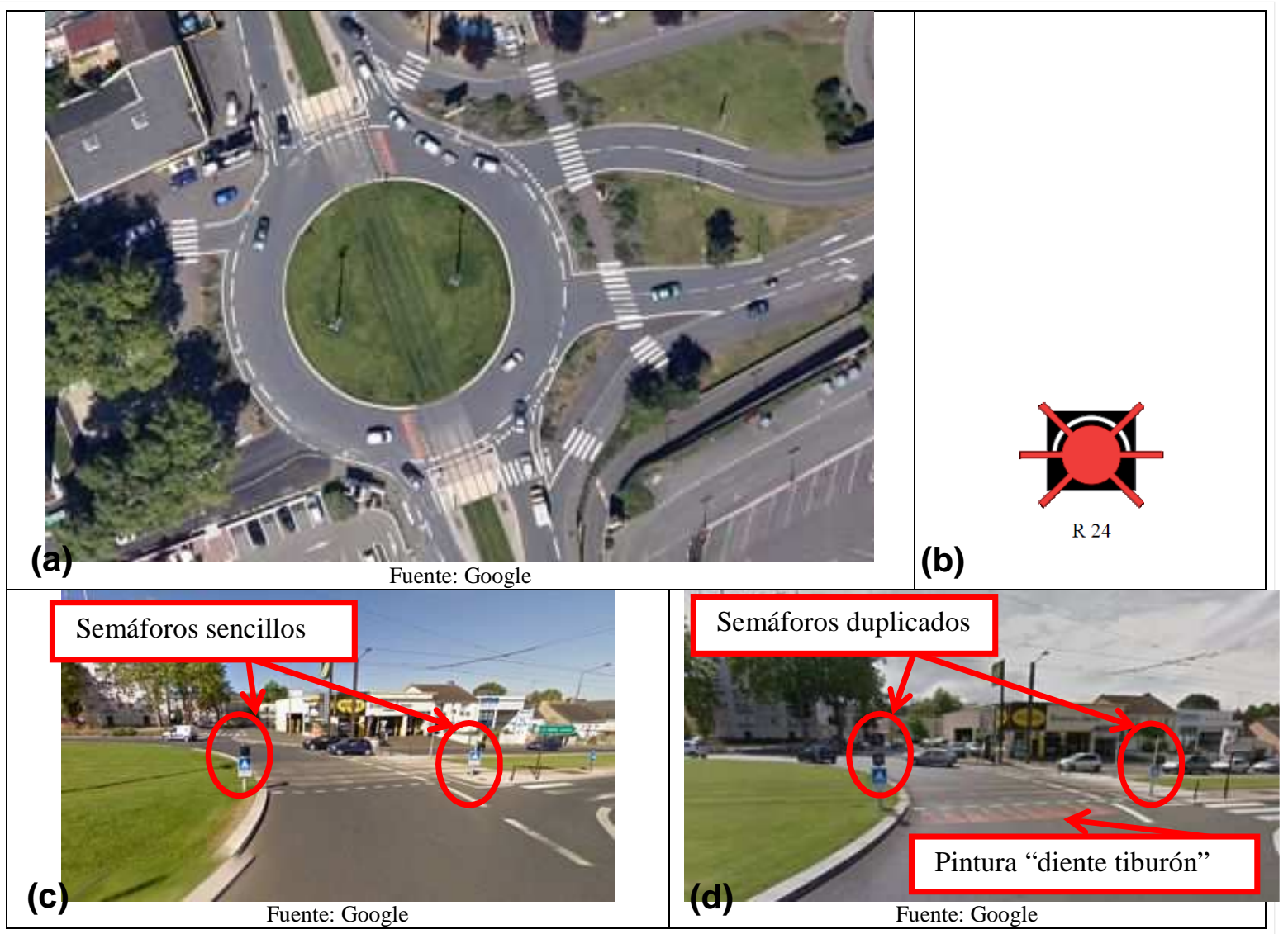

Fig. 7 - Antiguo punto conflictivo en la glorieta Paul Cézanne.

a) Disposición de la glorieta.

b) Semáforo de tipo R24

c) Situación antes de la implementación de las medidas.

d) Situación tras la implementación de las medidas.

\subsubsection{Glorieta São Brás, Oporto (Portugal)}

En este caso el sistema de metro ligero circula también por el centro de la glorieta de São Brás y se han producido varios accidentes por infracciones de los conductores de vehículos de carretera del tipo paso del semáforo en rojo (4 accidentes en un año).

Las medidas aplicadas para mejorar la situación fueron, en este caso (Figura 8):

- Mejora de la visibilidad de los semáforos mediante la recolocación de algunas señales que causaban confusión, y mediante el aumento del diámetro de la luz roja del semáforo para conseguir una mayor atención de los conductores.

- Mejora de la percepción mediante la disposición de nuevas líneas de parada pintadas en el pavimento. 
(a)
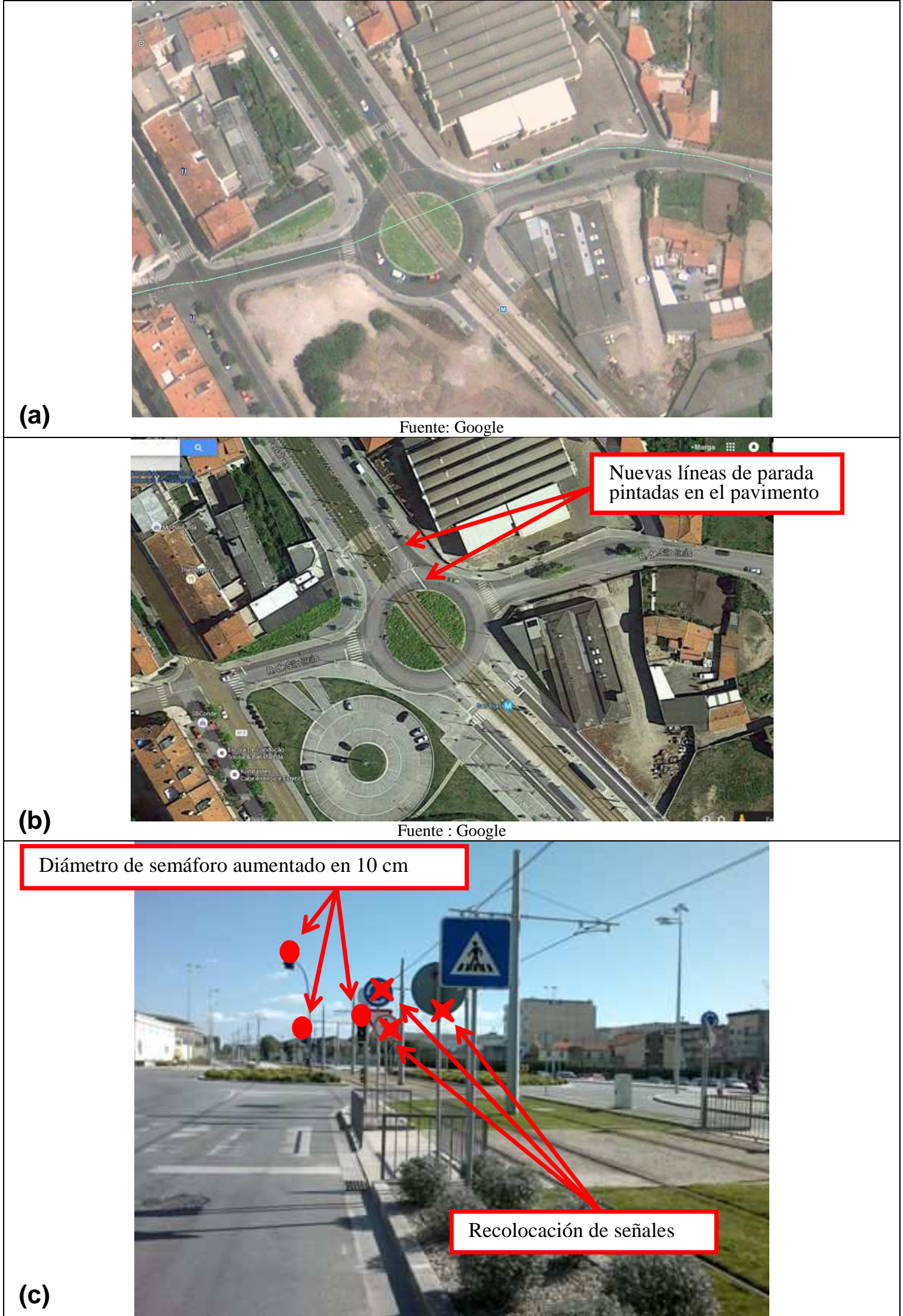

Fig. 8 - Antiguo punto conflictivo en la glorieta de São Brás.

a) Situación inicial de la glorieta.

b) Situación actual de la glorieta, con nuevas líneas de parada pintadas en el pavimento.

c) Medidas aplicadas en la entrada de la glorieta. 


\subsubsection{Glorieta Cruz de Piedra, Tenerife (España)}

La primera línea del metro ligero de Tenerife se inauguró en junio de 2007. Esta línea circula por el centro de la glorieta Cruz de Piedra. En este caso, como se muestra en la Figura 9a, dos de las entradas de las calles a la glorieta están muy cercanas a los puntos en los que el vial circular cruza las vías del metro ligero. Esto da lugar a una situación complicada, ya que los conductores de los vehículos de carretera tienen que fijar su atención en otros posibles vehículos llegando por su izquierda en el vial circular, pero tienen que cambiar inmediatamente su atención a los semáforos que protegen el cruce sobre las vías del metro ligero y a los vehículos de este sistema que pueden llegar tanto por la izquierda como por la derecha.

Debido a esta situación, se han producido varios accidentes por infracciones del tipo paso del semáforo en rojo por parte de los conductores. Las medidas aplicadas (en noviembre de 2007) se centraron en mejorar la protección, de la siguiente manera (Figuras 9c y 9d):

- Duplicación de los semáforos adyacentes a las vías de metro ligero: se dotó a los semáforos existentes con una nueva luz doble pequeña a la altura de los ojos de los conductores, para reforzar la atención de los mismos al llegar a la intersección.

- Implantación de nuevos semáforos en las entradas a la glorieta que están próximas al punto de cruce con las vías de metro ligero.

Adicionalmente se llevó a cabo una campaña de seguridad vial distribuyendo folletos entre los conductores de vehículos de carretera, recordando las normas de seguridad de la circulación. Incluso se patrocinó un programa de televisión sobre seguridad vial.

La evolución del número de accidentes y del número de frenados de emergencia (entre paréntesis) en este punto es el siguiente: junio a final de 2007: 9 (64); año 2008: 7 (24); 2009: 4 (29); 2010: 2 (14); 2011: 1 (9); 2012: 0 (11); 2013: 3 (12). De nuevo en este caso la reducción se puede deber al tiempo de aprendizaje del resto de usuarios del espacio urbano, mencionado anteriormente. En cualquier caso, parece que se ha producido una ligera mejora debido a las medidas implementadas, aunque los datos no son concluyentes. 


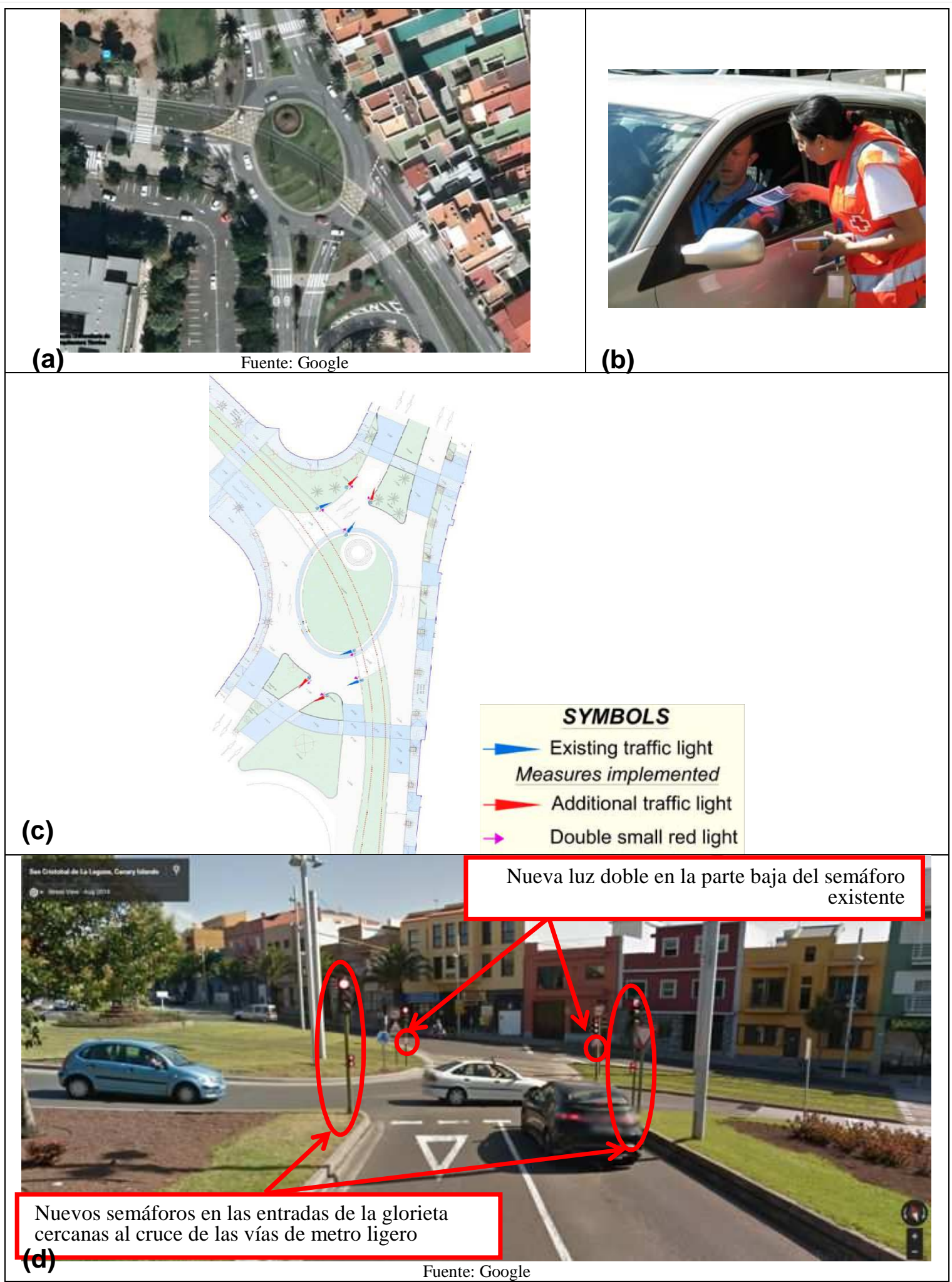

Fig. 9 - Antiguo punto conflictivo en la glorieta de Cruz de Piedra.

a) Disposición de la glorieta.

b) Campaña de seguridad vial.

c) Esquema de las medidas aplicadas (semáforos).

d) Foto de las medidas aplicadas (semáforos). 


\subsubsection{Glorieta Carretera Reial - Baix Lobregat, Barcelona (España)}

En este caso, el metro ligero circula por la glorieta en una alineación descentrada (Figura 10), generando una situación similar a la de un giro a la izquierda con problemas de visibilidad. La glorieta conecta con una autopista y la intensidad de tráfico es elevada. En este punto se han producido varios accidentes debidos a que los conductores de carretera se encontraban con el semáforo en verde para acceder a la glorieta (semáforo 1) pero a continuación tenían un semáforo en rojo para girar a la izquierda cruzando las vías del metro ligero (semáforo 2). El semáforo 1 en verde está pensado para los vehículos de carretera que continúan de frente, pero la consecuencia es que muchos conductores que querían girar a la izquierda entraban en la glorieta y no se esperaban encontrarse el semáforo 2 en rojo, dado que en las glorietas sin metro ligero el vehículo de carretera siempre tiene la prioridad una vez que ha entrado en el vial circular.

Las medidas aplicadas (durante el año 2007) fueron las siguientes (Figura 10):

- Mejora de la percepción (y aumento del tiempo de reacción en caso de situación de riesgo) mediante el desplazamiento de la línea de parada a una zona lo más alejada posible de las vías de metro ligero.

- Mejora de la protección mediante un cambio en el ciclo semafórico, poniendo en rojo los semáforos de todas las entradas a la glorieta cuando el metro ligero se está aproximando. Esta medida tiene el inconveniente de que empeora el flujo de tráfico de carretera y puede dar lugar a mayores niveles de congestión.

La evolución del número de accidentes en este punto es la siguiente: año 2006: 10 accidentes; año 2007: 8; 2010: 1; 2011: 2; 2012: 3; 2013: 1. Parece que se ha producido una mejora debido a las medidas implementadas. 


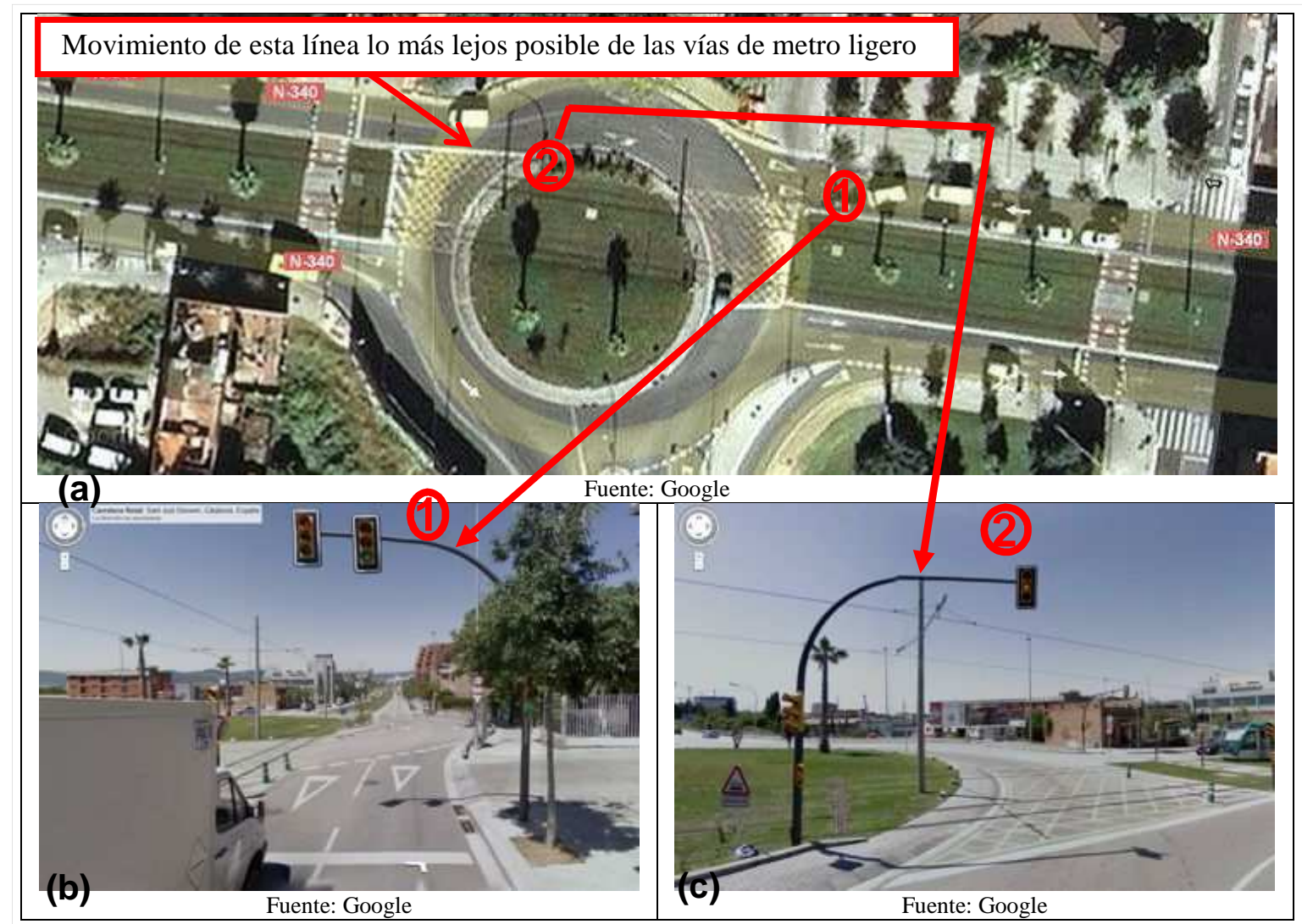

Fig. 10 - Antiguo punto conflictivo en la glorieta de Carretera Reial - Baix Lobregat. a, b y c) Disposición de la glorieta; recolocación de la línea de parada; y situación de los semáforos.

\subsubsection{Glorieta Ernest Granier, Montpellier (Francia)}

La línea 1 del metro ligero de Montpellier circulaba por el centro de esta glorieta a una velocidad de $40 \mathrm{~km} / \mathrm{h}$. Se produjeron varios incidentes debido a que los conductores de los vehículos de carretera no respetaban los semáforos, con un número total de 23 desde el año 2000 hasta el año 2009 (con 4 incidentes en 2009, 4 en 2008 y 5 en 2007).

La solución a este punto problemático se introdujo con la construcción de la nueva línea 3 (en el año 2010), conectada a la línea 1 en el centro de esta glorieta. Debido a la instalación del desvío de conexión se redujo la velocidad del metro ligero en esta zona a $15 \mathrm{~km} / \mathrm{h}$. Adicionalmente se cambió el funcionamiento de toda la intersección: se convirtió en una intersección convencional (aunque con una forma romboidal) con dirección única de circulación, dos carriles (más uno para giros a la derecha), y control de los cruces entre el metro ligero y los vehículos de carretera y de estos últimos entre ellos mediante semáforos.

Estos cambios dieron lugar a la ausencia de accidentes desde 2009. Se debe destacar que la solución fue cara, y que la reducción de la velocidad del metro ligero tiene consecuencias en la calidad del servicio. Por otra parte, dado que se introdujeron simultáneamente varios cambios (la modificación de la configuración de la intersección y la limitación de velocidad), no se puede determinar cuáles son los efectos de cada una de las medidas por separado. 


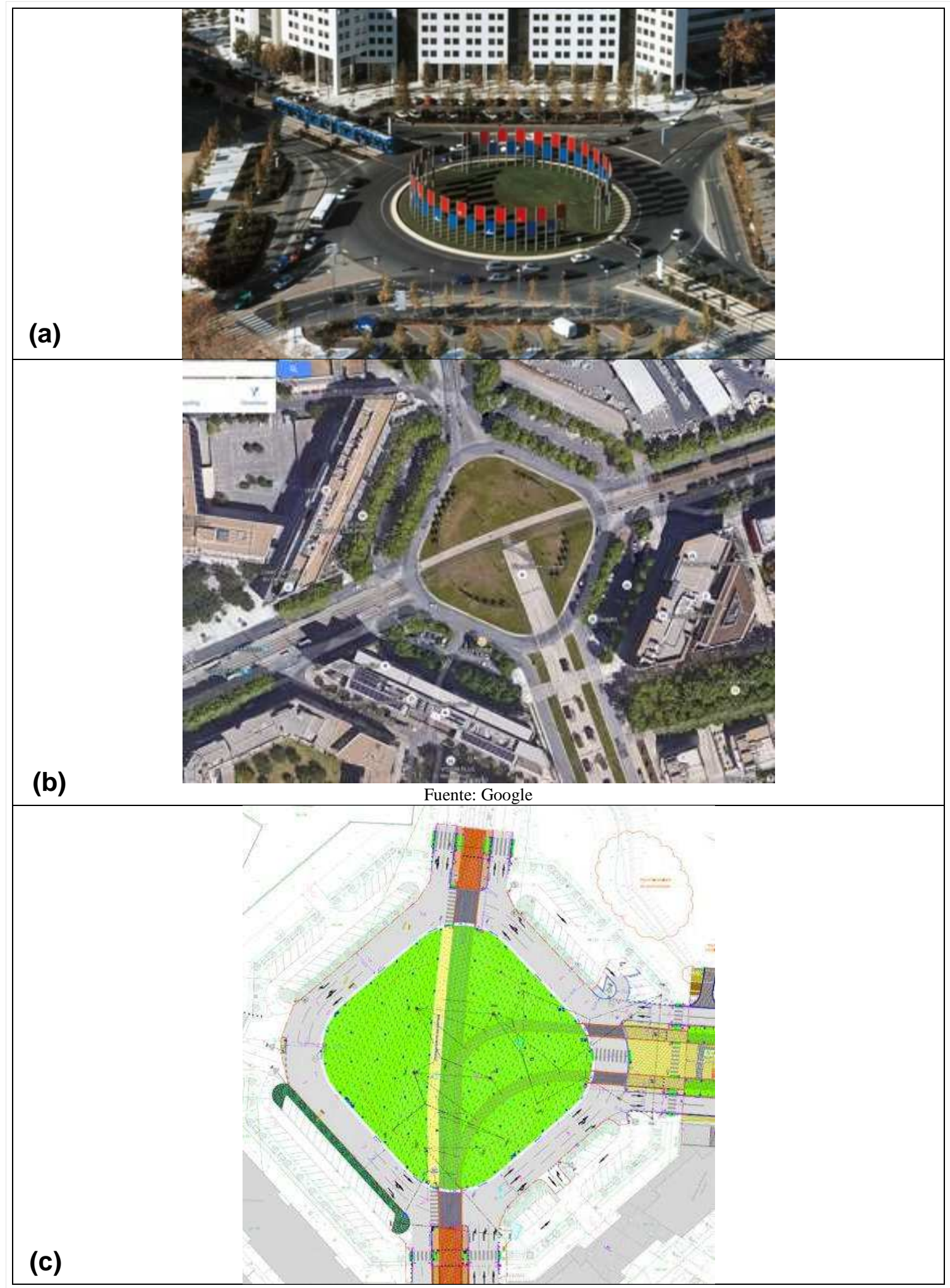

Fig. 11 - Antiguo punto conflictivo en la glorieta de Ernest Granier.

a) Situación inicial de la glorieta.

b) Situación actual como intersección convencional de forma romboidal.

c) Esquema de la situación actual de la intersección. 


\subsubsection{Otras medidas para mejorar la seguridad en las glorietas}

Algunas de las otras medidas mencionadas en el cuestionario sobre puntos de interacción, además de las presentadas previamente, son:

- Para mejorar la percepción: marcado de la isla central y del vial circular en diferentes colores, y resaltar la existencia de las vías y el área de barrido mediante la utilización de diferentes colores y materiales; y proporcionar señales informativas acerca del cruce de la glorieta por el metro ligero en las entradas de la misma.

\section{CONCLUSIONES}

En esta ponencia se han presentado algunos de los antiguos puntos conflictivos de redes de metro ligero cuya información se ha recopilado a través del cuestionario realizado durante la Acción COST TU 1103 "Operation and safety of tramways in interaction with public space". En este cuestionario participaron 24 operadores de metro ligero.

Los antiguos puntos conflictivos (hotspots) se han definido como los puntos de la red que solían tener un número de accidentes relativamente alto, pero que se han convertido en lugares más seguros con la aplicación de ciertas medidas. Para cada uno de los antiguos puntos conflictivos se han detallado las medidas implantadas para mejorar la situación. Estas medidas pueden ser aplicadas por otros operadores para resolver problemas similares, siempre teniendo en cuenta los condicionantes locales de cada red.

Se han incluido, cuando ha sido posible, datos sobre el número de accidentes anuales antes y después de la implementación de las medidas explicadas, para tratar de respaldar su eficiencia. Sin embargo, se debe destacar que, en general, las mejoras en estos datos son muy ligeras, porque afortunadamente el número de accidentes de los sistemas de metro ligero es normalmente bajo. Por otro lado, diversos factores pueden tener influencia en estos datos, como pueden ser los cambios en la intensidad de tráfico de carretera, nuevas limitaciones de velocidad, etc., de manera que resulta difícil inferir si las medidas han sido efectivas o no.

En cualquier caso, la causa principal de accidentes en los sistemas de metro ligero está en la interacción con otros usuarios del espacio urbano, debido al incumplimiento por parte de estos últimos de las normas de circulación, bien sea de forma voluntaria o involuntaria (en este último caso normalmente por la no percepción de las señales o semáforos, o por la confusión sobre cuáles les atañen).

Además del cuestionario sobre puntos conflictivos, durante la Acción se realizó otro sobre buenas y malas prácticas en el diseño de los puntos de interacción, recogiendo más de 130 ejemplos. En esta ponencia se ha incluido también un resumen muy somero sobre algunas de las conclusiones obtenidas a través de dicho cuestionario.

Durante la Acción COST se realizó un análisis minucioso de toda la información recopilada, que se ha sintetizado en el informe final, disponible en la página web de la Acción 
(http://www.tram-urban-safety.eu/). Una parte del informe se centra en los riesgos que se plantean en cada tipo de punto de interacción, y medidas para evitar dichos riesgos, con numerosos ejemplos reales, que puede resultar de interés para los lectores de esta ponencia.

\section{AGRADECIMIENTOS}

Los autores agradecen a la European Cooperation in Science and Technology (COST) la financiación de la COST Action 1103: "Operation and safety of tramways in interaction with public space”. Adicionalmente, los autores agradecen las aportaciones de las personas de las empresas operadoras de metro ligero que han cubierto los cuestionarios sobre puntos conflictivos y puntos de interacción. La redacción de esta ponencia no habría sido posible sin sus contribuciones.

\section{REFERENCIAS}

CERTU (2008). Giratoires et Tramways. Franchissement d'un carrefour giratoire par une ligne de tramways. Guide de Conception. CERTU (Centre d'études sur les réseaux, les transports, l'urbanisme et les constructions publiques). ISBN: 978-2-11-097155-5.

COIFMAN, B. y BERTINI, R.L. (1997). Median Light Rail Crossings: Accident Causation and Countermeasures. California PATH Working Paper UCB-ITS-PWP-97-13.

CURRIE, G. y REYNOLDS, J. (2011). Managing Trams and Traffic at Intersections with Hook Turns: Safety and Operational Impacts. Transportation Research Record, Vol. 2219, pp. 10-19.

FARRÁN, J.I. (2000). No Turns Allowed. Controlling Vehicles Turning in Front of Light Rail Vehicles. Transportation Research Record, Vol. 1704, pp. 85-89.

FONTAINE, L. (CHAIR), ABLASSER, G., BAKABA, J.E., BAPTISTA, J., BERTRAND, D., CARRASCO, N., CARSI, J., DE JONG, M., DEUTSCH, V., DUHOUX, M., GAIVOTO, C., GATTUSO, D., GOCH, K., IGNACCOLO, M., INTURRI, G., JASINSKI, M., KAMMACHI, A., KUPFERSCHMID, J., LANGENSIEPEN, D., LEWISCH, S., LOHRMANN, K.D., MACSINKA, K., MALASEK, J., MANSO, O., MARTI, C., MILLOT, M., MONTI, F., MORLEY, R., MUÑOZ, A., NAEGELI, L., NOVALES, M., ORTIZ, O., ROOS, R.J., ROSENBERGER, M., SCHMITT, D., SCHRÖTER, R., SCHWERTNER, M., SMIT, C., SPOUSTA, J., SUCHA, M., TEIXEIRA, M., TIERNEY, D., ULLMANN, M., VANA, M., VAN GENECHTEN, R., VASICEK, J., WALMSLEY, D., WEIDMANN, U., YEZBEK, J., YOUNG, T. y ZAIDEL, D. (2015). Operation and safety of tramways in interaction with public space. Analysis and outcomes. Detailed report. COST: European Cooperation in Science and Technology. ISBN: 978-2-11-139720-0.

KORBE, H.W., OGDEN, B.D., SIQUES, J.T., MANSEL, D.M., RICHARDS, H.A., GILBERT, S., BONI, E., BUTCHKO, M., SRUTTS, J.C. y HUGUES, R.G. (2001). TCRP Report 69: Light Rail Service: Pedestrian and Vehicular Safety. TRB (Transportation Research Board), Washington DC. ISBN 0-309-06704-9.

NOVALES, M., TEIXEIRA, M. y FONTAINE, L. (2014). LRT Urban Insertion and Safety: European Experiences. Transportation Research Record, Vol. 2419, pp. 63-81. 
PECHEUX, K.K. y GOLEMBIEWSKI, G.A. (2011). Driver Comprehension and Assessment of Traffic Control Devices at Signalized Intersections Interfacing with Light Rail Transit. Transportation Research Record, Vol. 2219, pp. 1-9.

PECHEUX, K.K. y SAPORTA, H. (2009). TCRP Synthesis 79: Light Rail Vehicle Collisions with Vehicles at Signalized Intersections. TRB (Transportation Research Board), Washington DC. ISBN 978-0-309-09821-2.

TYDLACKA, J., VOIGT, A.P., LANGFORD III, W.C. (2011). Evaluation of Lighted Pavement Marker Stop Bars at Intersections with Light Rail Lines. Transportation Research Record, Vol. 2250, pp. 49-56. 